\title{
CT IMAGING OF SMALL ANIMALS USING MONOCHROMATIZED SYNCHROTRON X RAYS
}

\author{
F.A. Dilmanian ${ }^{1}$, H. Rarback ${ }^{1}$, E. Nachaliel ${ }^{1}$, M. Rivers ${ }^{1}$, W.C. Thomlinson ${ }^{1}$, R. Appel ${ }^{4}$, \\ L.D. Chapman ${ }^{1}$, R.F. Garrett ${ }^{2}$, P.N. Luke ${ }^{3}$, M.H. Miller ${ }^{4}$, R. Pehl ${ }^{3}$, T. Uversluizen ${ }^{1}$, D.N. Slatkin ${ }^{1}$, \\ P. Spanne ${ }^{1}$, S. Spector ${ }^{1}$, and A.C. Thompson ${ }^{3}$
${ }^{1}$ Brookhaven National Laboratory, Upton, NY
${ }^{2}$ Australian Nucl. Sci, and Tech. Organization, Sidney, Australia
${ }^{3}$ Lawrence Berkeley Laboratory, Berkeley, CA
${ }^{4}$ Analogic Corporation, Peabody, MA

\section{Abstract}

Rats and chicken embryos were imaged in vivo with a prototype Multiple Energy Computed Tomography (MECT) system using monochromatized $x$ rays from the $X 17$ superconducting wiggler at the National Synchrotron Light Source (NSLS). The CT comfiguration consisted of a horizontal, low-divergence, fan-shaped beam, $70 \mathrm{~mm}$ wide and $0.5 \mathrm{~mm}$ bigh, and a subject rotating about a vertical axis. A linear-array high-purity $\mathrm{Ge}$ detector with 140 elements, each $0.5 \mathrm{~mm}$ wide and $6 \mathrm{~mm}$ thick, was used with a data acquisition system that provides a linear response over almost six orders of magnitude of detector current. The dual photon absorptiometry (DPA) algorithm was applied to images of the rat head acquired at 20 and $45 \mathrm{keV}$ to obtain two new images, one representing the low- $Z$, and the other the intermediate- $Z$ element group. The results indicate that the contrast resolution and the quantification accuracy of the images improve stepwise; first, with the monochromatic beam and, second, the DPA method. The system is a prototype for a clinical brain scanner.

\section{INTRODUCTION}

We report the results of the first animal studies with the prototype MECT $[1,2]$ system developed with a field-of-view (FOV) of $65 \mathrm{~mm}$. The work extends the first phantom studies with the system reported earlier $[3,4]$. The clinical MECT system, which is expected to be ready for neuroradiology in late 1994, will have an FOV of $20 \mathrm{~cm}$. The goals of the MECT project are a) to demonstrate the performance of a monochromatic CT (i.e., a CT with an energy bandwidth $<0.5 \%$ ), and b) to apply the system to clinical research problems. The monochromaticity of the beam not only eliminates beam hardening effects, but also allows efficient use of the energy selective methods of DPA and $\mathrm{K}$-edge subtraction (KES) of iodine and other contrast elements. The results are good contrast resolution and accurate quantification of the image.

\section{EXPERIMENTAL METHOD}

The experiments were carried out at the B1 section of the $\mathrm{X} 17$ iamline, with the wiggler operating at 4.6 tesla. The synchrotron $\mathrm{x}$ ray beam, filtered only with the beamline Be windows $(1.8 \mathrm{~mm}$ thick) and a carbon foil $(0.39 \mathrm{~mm}$ thick), was collimated to $70 \mathrm{~mm}$ horizontal width and $1 \mathrm{~mm}$ vertical width, and then monochromatized: The system components are shown below.

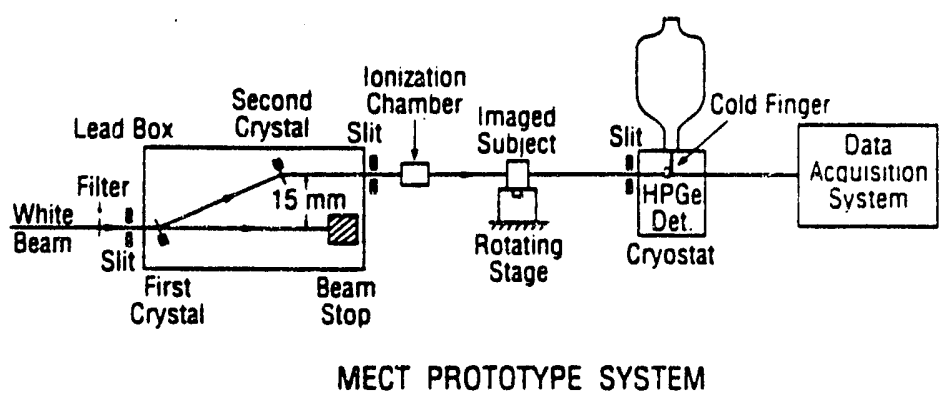

Fig. 1 Schematic view of the prototype MECT system.

\section{A. The Monochromator}

The monochromator, a two-crystal fixed-exit tunable Laue-Laue device providing an energy range of $20-92 \mathrm{keV}$ when equipped with $\mathrm{Si}<220>$ crystals, was described elsewhere [5]. It was developed to serve the prototype MECT system as well as the Materials Science research program at X17. Each $\mathrm{Si}<220>$ crystal is $75 \mathrm{~mm}$ wide, 7 mm high, and $1 \mathrm{~mm}$ thick. The heat load on the first crystal is about 100 watts. The crystal was water-cooled using a molybdenum cooling block thermally coupled to the widened base of the Si crystal with In-Ga liquid metal. Two electrostrictive devices [6], one of $30 \mu \mathrm{m}$ and the other of $105 \mu \mathrm{m}$ stroke, were used for angular positioning of the second crystal in the Bragg and the tilt directions, respectively. Measurements showed less than $20 \%$ variation in beam intensity along the $70-\mathrm{mm}$ horizontal beam width at a detuning angle corresponding to a monochromator yield of $6 \%$ of the peak. The monochromator was detuned to suppress harmonics [7]. The noise on the monochromatic beam caused by the vibrations of the crystals was reduced to $2 \% \mathrm{rms}$ by reinforcing the monochromator's stand and removing major sources of vibration (i.e., motors and fans) from the room.

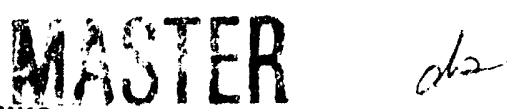




\section{DISCLAIMER}

This report was prepared as an account of work sponsored by an agency of the United States Government. Neither the United States Government nor any agency thereof, nor any of their employees, makes any warranty, express or implied, or assumes any legal liability or responsibility for the accuracy, completeness, or usefulness of any information, apparatus, product, or process disclosed, or represents that its use would not infringe privately owned rights. Reference herein to any specific commercial product, process, or service by trade name, trademark, manufacturer, or otherwise does not necessarily constitute or imply its endorsement, recommendation, or favoring by the United States Government or any agency thereof. The views and opinions of authors expressed herein do not necessarily state or reflect those of the United States Government or any agency thereof. 


\section{B. The Detector}

A 140-element, high-purity Ge linear-array detector, developed for this program by Lawrence Berkeley Laboratory [8], was used. The individual elements of the detector were $0.4 \mathrm{~mm}$ wide $(0.5 \mathrm{~mm}$ center-to-center spacing), $10 \mathrm{~mm}$ high, and $6 \mathrm{~mm}$ thick.

\section{The data acquisition system}

The data acquisition system (DAS), operating in a current-filtration mode, was designed and constructed by Analogic Corp, Peabody, MA. The system amplifies the current from each detector element, filters the signal and digitizes it. Signals are detected with a large dynamic range (close to $10^{6}: 1$ ) and an integral linearity of $\pm 0.03 \%$. Each group of 128 channels is multiplexed to use a single precision amplifier in which the gain is switched dynamically between three levels, differing from each other by factor of 8 , and a single 16-bit ADC. Consequently, a 14-bit precision word is produced over a 20-bit dynamic range (the two least significant bits of the ADC were not used).

The interface electronics were primarily based on CAMAC electronics. A VAXstation 3100 model 76 was used for data acquisition and image reconstruction. The acquisition system consists of a dedicated CAMAC memory to receive the DAS output, a multichannel scaler to record the position of the rotary stage and the phase of the AC line (needed for noise suppression) for each view, and CAMAC motor drivers to run the subject apparatus stages, the beamline apertures, and the monochromator. The acquisition hardware was designed so that no computer intervention is needed on a view-by-view basis. The raw data are available in near-real time so that the operator can adjust the acquisition parameters. Interactive Data Language (IDL) [9] was used for both data acquisition and processing.

\section{The apparatus for animal positioning}

The animal positioning system included (in the order from bottom to top): a) a high-precision elevator with a 20 $\mathrm{cm}$ travel range to adjust the height of the subject in the beam; b) a 'cradle' with two mutually perpendicular rotation axes, both in the horizontal plane, to align the plane of rotation parallel to the plane of the fan beam; c) a linear translator that moves the rotating stage, that rotates the subject being imaged, laterally to the beam; d) the rotator; and e) two linear translators with mutually perpendicular axes, both in the horizontal plane, that held the animal holder. All the stages were driven by stepping motors. The entire system was placed on a table with adjustable height. The system was used to align the axis of rotation orthogonal to the plane of the fan beam within about one arc-minute.

\section{EXPERIMENTAL RESULTS}

Rats were anesthetized by a single injection of ketaminexylasine, and positioned in a plastic bottle at an upward angle of about $50^{\circ}$ to the horizontal plane (Fig. 2). They were imaged in one or more planes through the cerebrum (upper head), using the DPA method at 20 and $45 \mathrm{keV}$. The image acquisition was carried out at a viewing rate of $120 \mathrm{~Hz}$, using an angular speed of $360^{\circ}$ in 8 seconds for the rotating stage (i.e., and angular step of $0.375^{\circ}$ ).

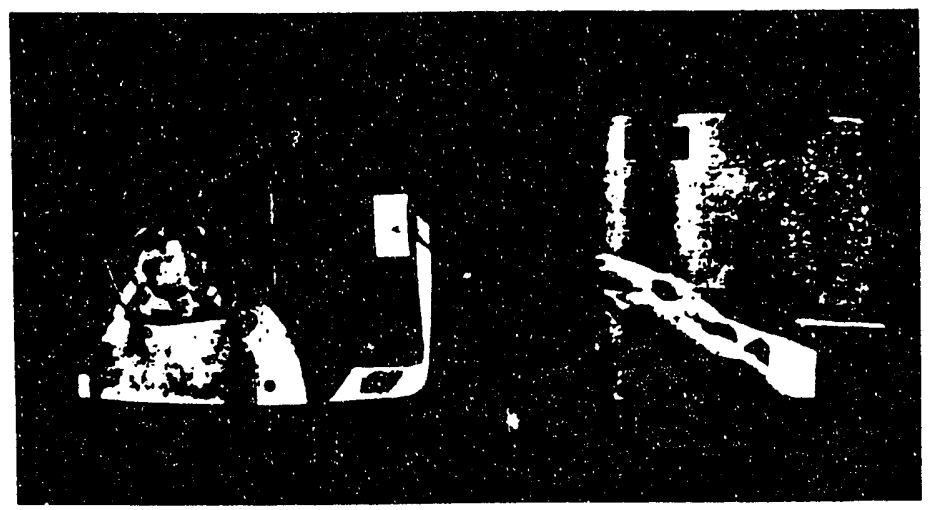

Fig. 2 The lateral and top view of the rat positioned for imaging. The horizontal line on the lateral view indicates the length of the slice imaged.

For each slice and for each energy, two sets of CT images were obtained, with the rotation axis in the second set moved laterally half the spacing of the detector elements (i.e., $0.25 \mathrm{~mm}$ ) with respect to the first set. The results were combined in a single reconstruction calculation, in which pixels from the two sets of projections were interlaced to produce a new set of projections with a pixel size which was half of that in the original projections. In this way, an inplane spatial resolution of about $0.3 \mathrm{~mm}$ FWHM was obtained.

Figs. 3 and 4 show the $20 \mathrm{keV}$ and $45 \mathrm{keV}$ images, while Figs. 5 and 6 show the low- $Z$ and the high- $Z$ images obtained by applying the DPA algorithm to the results. The pixel size in the images is $0.125 \times 0.125 \mu \mathrm{m}^{2}$.

\section{CONCLUSIONS}

The results indicate that the DPA method enhances the image contrast resolution in the bone (in the high- $Z$ image), and in the soft tissue (in the low- $Z$ image), although image artifacts caused by poor detector channels and imperfect registration of the low- and high-energy images partially obscure the effect, especially in the low- $Z$ image. Assuming that the single-energy images (Figs. 3 and 4 ) have more contrast resolution than they would with bremsstrahlung radiation, the improvement in the image contrast resolution seen in Figs. 5 and 6 come not only from the monochromatized beam, but also from the use of the DPA method. The contrast resolution and the accuracy of quantification of the images, however, are still far below the values set for the final goals of the project. 


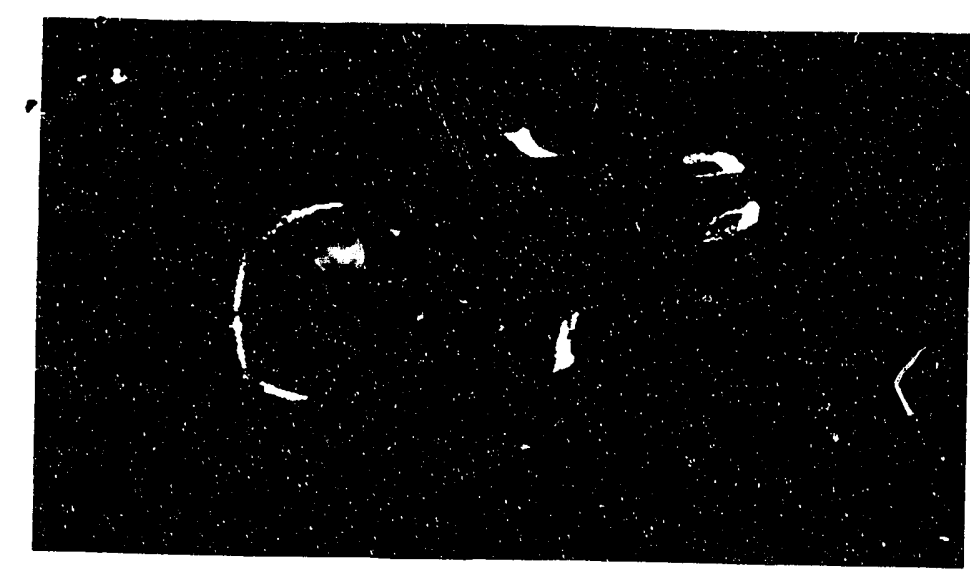

Fig. 3 20-keV image.

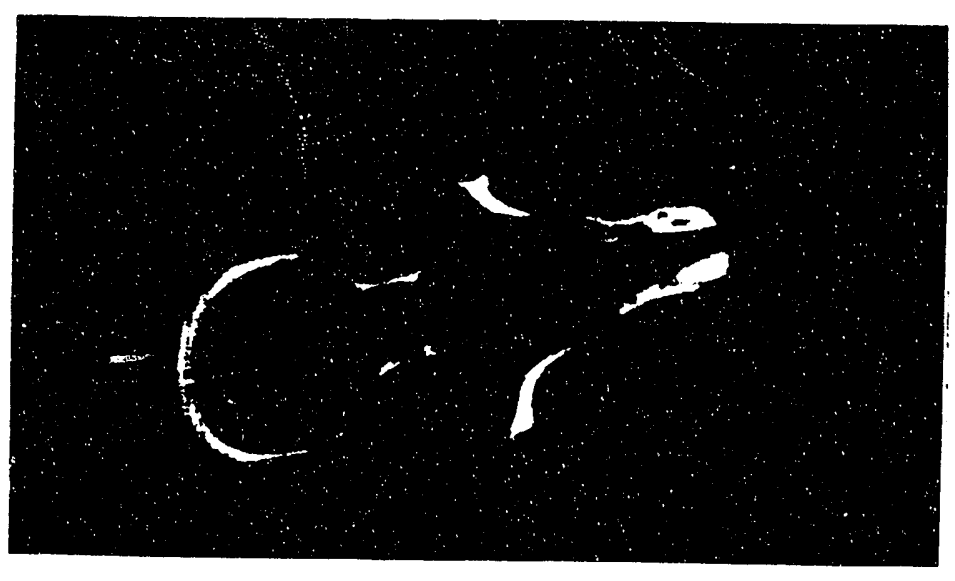

Fig. $4 \quad 45-\mathrm{keV}$ image.

\section{ACKNOWLEDGEMENTS}

We thank L.E. Berman, J.B. Hastings, S.K. Sharma, D.P. Siddons, and P.M. Stefan for their help in design of the monochromator; and C. Brite, N.F. Gmür, R. Greene, N. Lazarz, A. Lenhard, A. Levian, P. Micca, M. Nawrocky, M. Shleifer, and K. Vodopia for their technical support. We also thank A.D. Woodhead for critical review of the manuscript, and A.L. Ruggiero for preparation of the manuscript. This research was supported by U.S. DOE contract DE-AC-02-76CH00015.

\section{REFERENCES}

[1] FA. Dilmanian, R.F. Garrett, W.C. Thomlinson, L.E. Berman, L.D. Chapman, N.F. Gmür, N.M. Lazarz, P.N. Luke, H.R. Moulin, T. Oversluizen, D.N. Slatkin, V. Stojanoff, A.C. Thompson, N.D. Volkow, and H.D. Zeman. Physica Medica VI, 301-307, 1990.

[2] F.A. Dilmanian, R.F. Garrett, W.C. Thomlinson L.E. Berman, L.D. Chapman, J.B. Hastings, P.N. Luke, T. Oversluizen, D.P. Siddons, D.N. Slatkin, V. Stojanoff, A.C. Thompson, N.D. Volkow, and H.D. Zeman. Nucl. Instr. and Meth. in Phys. Res. B56/57, 1208-1213, 1991.

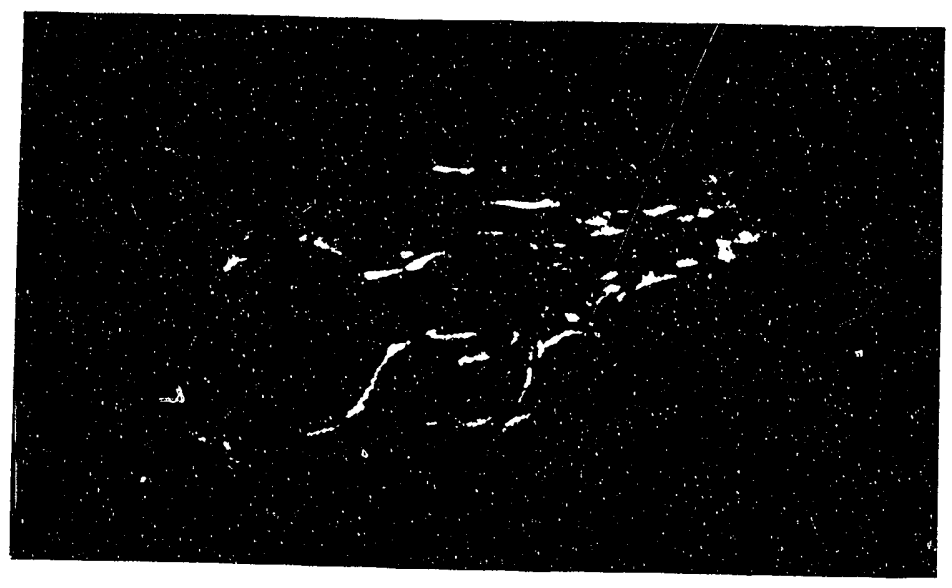

Fig. 5 Low-Z image.

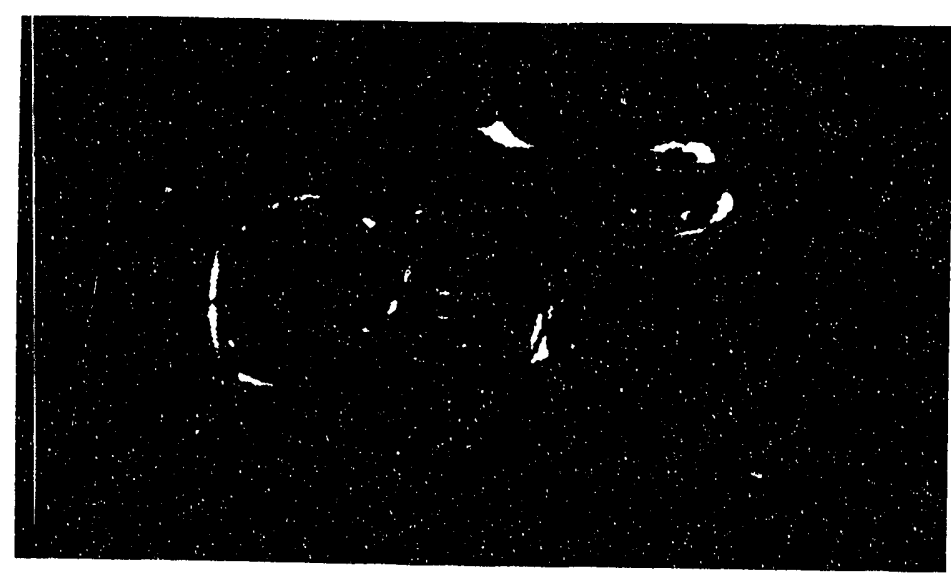

Fig. 6 Intermediate- $Z$ image.

[3] E. Nachaliel, F.A. Dilmanian, R.F. Garrett, W.C. Thomlinson, L.D. Chapman, N.F. Gmür, N.M. Lazarz, H.R. Moulin, M.L. Rivers, H. Rarback, P.M. Stefan, and P. Spanne. Nucl. Instr. and Meth. in Phys. Res. A319, 305-310, 1992.

[4] F.A. Dilmanian, E. Nachaliel, R.F. Garrett, W.C. Thomlinson, L.D. Chapman, H.R. Moulin, T. Oversluizen, H.M. Rarback, M. Rivers, P. Spanne, A.C. Thompson, and H.D. Zeman. Conference record of the 1991 IEEE Nucl. Sci. Symp. and Med. Imag. Conf. Vol. 3, 1831-1834, 1991.

[5] R.F. Garrett, F.A. Dilmanian, T. Oversluizen, A. Lenhard, L.E. Berman, L.D. Chapman, and W. Stoeber. Rev. Sci. Instrum. 63, 595-598, 1992.

[6] B. Rawal and J. Galvagni. Motion Control, pp 29-33, January 1992.

[7] M. Hart and A.R.D. Rodrigues, J. Appl. Cryst. 11, 248253, 1978.

[8] P.N. Luke, IEEE Trans. Nucl. Sci. NS-31, 312-315, 1984.

[9] IDL, Research Systems, Inc., Boulder, Colorado. 

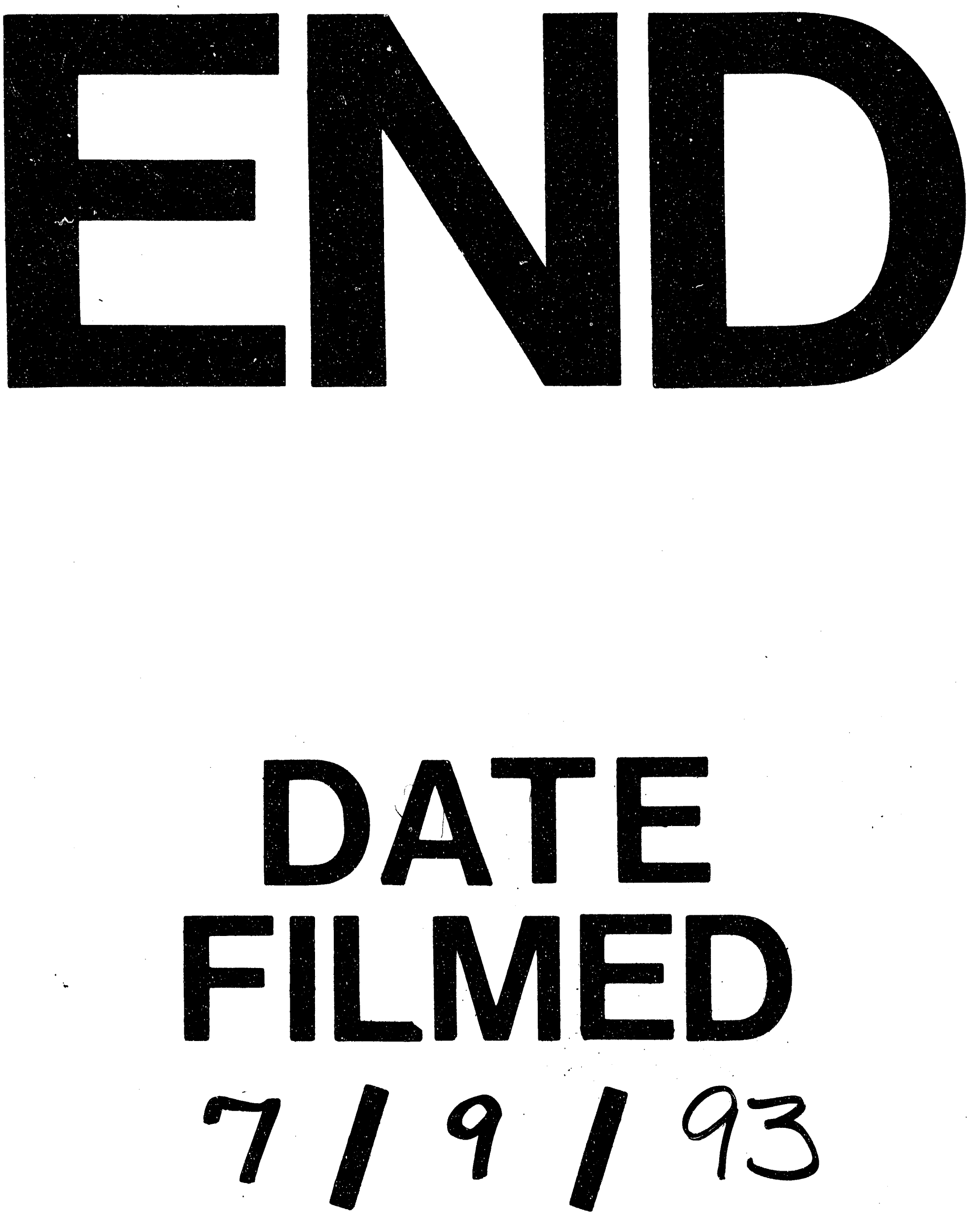

1 
$i$ 